\title{
Chapter 2 \\ The Emergence of Written Language: \\ From Numeracy to Literacy
}

"HUMANKIND IS DEFINED BY LANGUAGE; but civilization is defined by writing. Writing made historical records possible, and writing was the basis for the urban societies of the old World."

- Peter T. Daniels (1996, p. 1)

"Cognitively as well as sociologically, writing underpins 'civilization', the culture of cities.”

- Jack Goody (1987, p. 300)

\begin{abstract}
This chapter reviews how written signs first emerged and developed into systematic writing systems. The first sign system appeared to fulfill accounting purposes for the preservation of private properties in antiquity. Initial written signs, including plain tallies, complex tokens, and tokens in clay envelopes, are reviewed. Written signs before the emergence of the Greek alphabet, such as cuneiforms and hieroglyphs, are also reviewed. As agricultural culture and urbanization took place, writing systems became more multifaceted and systematized. The characteristics of true alphabets are discussed. For a comparison purpose, the Chinese writing system is briefly mentioned. The chapter ends with a discussion of the transition from numeracy to literacy.
\end{abstract}

Keywords initial written signs · Cuneiforms · Hieroglyphs · Greek alphabet · Chinese writing systems $\cdot$ from numeracy to literacy

As the two epigraphs show, there is an indispensable relationship among human beings, language, and writing in terms of civilization. Especially, writing was the enabler and propeller of human civilization. Writing paradoxically did not evolve from oral language. It stemmed from the need of notational schemes for bookkeeping and commercial transactions using materials available in the surroundings 
(Logan, 2004). Hence, it is viewed not as a deliberate invention but as an incidental offspring of a strong sense of private property. Since quantitative information is much more difficult to remember than tales or legends, due to no storylines, characters, and plots involved in tallies, notational systems emerged to overcome our limited memory span (Logan, 2004). Once it was systemically adopted for use, the token system rapidly evolved and changed the way in which information was presented, transferred, and stored. The token-based notational system evolved to the numeric system, and further laid a foundation for more sophisticated notational systems-that is, writing systems.

Everything waxes and wanes over time. Natural selection takes shape longitudinally. Some signs quickly or slowly died out, while others endured for more than 3000 years (e.g., Chinese characters). No matter where and how early signs emerged, the effort to establish systematic notational systems shows our ancestors' delicate intelligence. It was writing that drastically extended humans' cognitive capacities. Writing exerted a considerable impact on at least three areas which were not mutually exclusive. First, human efforts to preserve private property led to the development of a concept that symbols could be used to communicate one another beyond the limited temporality (time) and locality (space) of oral communication. The sign systems began to serve as a means of communication that was not restricted to "real time" and could be stored accurately by overcoming the constraints of our memory and avoiding (possible) misinterpretations. Second, written signs showed a possibility that an abstract concept (e.g., numbers) could be represented through a symbolic medium. Writing symbolically represented both concrete objects (e.g., grains, crops, or livestock) and abstract concepts (e.g., numbers) through knots in strings and marked lines on clay tokens, stones, or turtle shells. These graphic systems not only facilitated the abstraction of objects and concepts, but also promoted the understanding of conceptual relations between concrete objects and abstract signs. Third, the notion of the one-to-one correspondence in encoding between symbols and meanings gave rise to the sound-symbol correspondence in the writing system. These elements incubated the potential for the development of systematic writing systems.

Within this context, this chapter reviews the evolution of writing systems. It first overviews pristine notational systems and important milestones in the evolution of writing. The large portion of the chapter is devoted to the early forms of writing. In order to stay focused on the linear progression of writing in a global sense, the different trajectories of all writings on the globe are not reviewed in this chapter. It is virtually impossible to review the specifics of all writing systems in one chapter. The purpose is to survey the evolution of writing until the point of the advent of the alphabet and Chinese characters. 


\subsection{Initial Written Signs}

\subsubsection{Plain Tallies}

A notational system for accounting purposes dates back to the period of 10,000 B.C. across many parts of the ancient world, while logograms and abstract numbers emerged approximately 5,000 years ago (3,000 B.C.). Early notational devices were made up of tallies in the forms of notched sticks, knotted strings, and etches on animal bones and shells (Logan, 2004). Although they did not carry intensive information, such as people involved in transactions, tallies demonstrated three significant cognitive implications. First, tallies showed the potential to visualize the quantitative concepts by means of concrete materials to save counting information, allowing for the storage of data so that users could rise above the ephemeral nature of the temporal moment. Second, tallies objectified the concept so that people involved in the transaction could understand the recorded content without confusion. Third, verbal information was transformed to nonverbal signs through the use of a metaphoric medium.

Although tallies fulfilled their primary purposes, the rudimentary system bore some limitations. Tallies were too simple to carry qualitative information associated with them, such as who did what and when in what context. Another limitation was that tallies could not indicate complex transactions. These limitations drove to more complicated recording devices.

\subsubsection{Complex Tokens}

The first tally system eventually increased in complexity, yielding the development of clay accounting tokens. As agriculture developed further, diverse staples, products, and commodities became available, which necessitated a more complex enumeration and accounting system. The concept of the unit of measurement started to develop accordingly.

The token system drastically changed data processing because mathematical concepts could be understood out of context by others and be translated into unambiguous tactile notions. With more meaningful and complex tokens, users were able to process a large amount of data simultaneously. They were also able to deal with different commodities concurrently through diversification. It further allowed for the classification of goods through organizing and reorganizing them into categories to fulfill book-keeping purposes. Logan (2004) noted that "the token system [was] the forerunner of the abacus as well as spreadsheet analysis" (p. 13).

The mathematical notation of tallies required at least three principles: (1) simplicity, (2) a one-to-one correspondence between an item and its corresponding mark, and (3) objectivity (Logan, 2004; Sampson, 2015). First, the recording system should not be complex so that everyone involved in transactions could easily 
understand the meaning of marks or tokens for the transparency of transactions. As a man-made artifact for accounting, tokens were gradually shaped into an organized marking system such that the system was easy to recognize and simple enough to be systematically reproduced. This was achieved by assigning each token to a discrete referent. Second, the principle of simplicity was achieved through the symbolic invention under the principle of a one-to-one correspondence. This meant that each mark had uniqueness for an independent meaning to avoid ambiguity. Through the one-to-one correspondence, it could be recognized and understood by all members involved in the commercial activities. Last, the notational system should also be objective. The objectivity was facilitated by the standardization of each sign. Early notational systems associated with goods or staples were quickly developed to satisfy these three conditions.

\subsubsection{Tokens in Clay Envelopes and More}

As more complex transactions frequently occurred, in need was a collective means that could be used as a receipt or a consolidated tool containing all tokens involved in a single transaction. Clay envelopes emerged to serve that purpose. Over the course of the token system development, clay envelopes at first functioned as containers of a number of plain tokens, as tokens were stored in a group within a sealed clay envelope. Since the envelope was made of clay, the content was not visible. The lack of visibility of contents in clay balls and envelopes prompted an effort to overcome the drawback for efficiency. Early accountants pressed numeric information or tokens on the wet and soft surface of the envelope in the form of a stylus cut in order to indicate the content of an envelope and transactional specifics involved in multiple transactions. The surface was also marked with the seals of individuals involved in the transactions. Therefore, it functioned as a receipt of a transaction and a documentation of contracts or agreements.

Notably, these impressed signs made the envelope carry redundant information, due to the duplicate data indicated in both inside (i.e., the number of tokens stored inside the envelope) and the outside surface of the envelope (i.e., what was written outside of the envelope). The curved envelopes quickly progressed to twodimensional flat tablets with impressed tokens on the surface. The evolution of envelopes into tablets marked a major breakthrough of information transmission and processing. This was a harbinger of creating written signs that represented tokens, paving the way for a major quantum leap to the creation of written signs that captured the notion of speech-sound and word-meaning relationships as a major step of abstraction. Eventually, the flat tablet became the prototype of all twodimensional writing surfaces we use these days, including the pages of a book, the display monitor of a computer, and digital tablets that are currently in use.

Early writing accelerated the development of metaphorical thinking and created a perceptual, cognitive, and mental condition that was conducive to the invention of more sophisticated writing systems (Logan, 2004; Wolf, 2007). This evolution 
resulted in the development of abstract numerals as quantitative notations and written words as qualitative records, leading to a crucial change of concepts. Clay tokens, such as cylinders, pyramids, discs, and balls, became systematized such that the signs could serve as a means of communication for universal use. In other words, tokens were further developed to convey meaning that individuals from different sociocultural communities could understand, regardless of the language they spoke.

The mode of abstraction motivated users to develop more sophisticated and complex systems that accommodated unmet needs with the rudimentary notational system. Through transforming verbal information to nonverbal data, the use of tallies and accounting systems gradually exerted a remarkable impact on human cognition and the human mind. The notational system reinforced the use of hand-eye coordination and fine motor skills essential for writing (Logan, 2004; Sampson, 2015). Token markings required the uniformity of shapes across recording signs and repeatable symbols as well as the arrangement of signs in a way that the user (or the reader) could grasp the whole field of data at a single glance. The volume, concreteness, and tactility of three-dimensional objects were lost with the two-dimensional tablet. The increased abstraction resulting from transferring three-dimensional data to two-dimensional tokens promoted the user's observational skills at a single glimpse. The visual thinking or acuity reinforced the skills of classification, analysis, uniformity, repeatability, and the power of the visual (Logan, 2004).

In summary, the development and enrichment of the token system and writing comprised largely five linear sequential stages: (1) plain tokens such as knotted strings and wedge-shaped marks, (2) complex tokens such as carved animal bones, (3) tokens stored in clay envelopes, (4) impressed logograms on clay envelopes containing tokens inside so that both interior and exterior could bear notational information, and (5) impressed logographs on two-dimensional clay tablets (Logan, 2004). Each stage established an important milestone for the emergence of cuneiforms, hieroglyphs, the early forms of alphabets, and finally the alphabet. A brief review of cuneiforms and hieroglyphs as precedents of the alphabet is in order.

\subsection{The Origin of the Alphabet}

Although they served as receipts of transactions and documentations of contracts, as stated earlier, clay envelopes had a shortcoming that contents were not visible. To overcome the disadvantage of being opaque, our ancestors impressed tokens onto the wet surface of the envelope. This technique allowed them to see the contents without breaking the envelope. This essentially resulted in the "unexpected and certainly unplanned side effect of creating the first two-dimensional logograms" (Logan, 2004, p. 19), which were two-dimensional visual signs that abstractly represented the written form of single words. This transformation yielded chain reactions that led to the invention of logographic writing, phonetic coding, and abstract numerals. It pushed a shift away from the use of the tactile sense (i.e., threedimensional tokens) to the more abstract visual sense (i.e., two-dimensional written 
signs). This established a sound basis for more developed written signs such as cuneiforms and hieroglyphs.

Although pictograms are considered the forerunners of writing, pictography does not qualify as writing (Daniels, 1996) because it does not entail specific linguistic forms. Daniels (1996) defines writing as "a system of more or less permanent marks used to represent an utterance in such a way that it can be recovered more or less exactly without the intervention of the utterer" (emphasis in original; p. 3). Based on this definition, writing is essentially connected to spoken language. This is consistent with Perfetti and Liu's (2005) notion that writing encodes spoken language. Daniels (1996) continues to claim that "each type of script entails about the same amount of effort to record the same amount of information" (p. 26). Although alternative classifications are possible, the tripartite classification of logography, syllabary, and alphabet remains the most popular (Daniels, 1996). As the alphabet is the most widely used script, the road to the advent of the alphabet is briefly reviewed below.

\subsubsection{Cuneiforms}

Around the fourth millennium B.C. (3300-3200 B.C.), an advanced form of writing emerged by Sumerians in lower Mesopotamia situated between the Tigris and Euphrates rivers (now Southern Iraq). Cuneiforms in wedge-shaped marks etched into clay tablets could inform us of a complex civilization established about 5,000 years ago. The word cuneiform was derived from the Latin word cuneus, meaning nail, which referred to the script's wedge-like appearance (Wolf, 2007). Given that the token system for accounting purposes led to the advent of the Sumerian writing system, the record of counting became the precursor of writing in Mesopotamia. As the society grew more complex, a more centralized administration was necessary. Since writing was useful for proper record-keeping, the use of writing for administrative purposes expedited the development of writing.

Archaic Sumerian writing used limited vocabulary that was context-bound. This was not sufficient enough to express sophisticated information, such as the quantities of material objects, people involved in transactions, and the units of measurement (Sampson, 2015). As such, the Sumerian cuneiform gradually lost pictographic elements and became more abstract and sophisticated by gaining more logographic elements. The logographic system at the time conveyed the meanings of objects and concepts through writing, but did not represent the sound of oral language in writing. Powell (1981) called the early Sumerian signs "mnemonic" (p. 421). However, Sampson (2015) is not in agreement with him on the reference to the mnemonic sign because "... archaic Sumerian writing appears to be a genuine writing system, of the logographic type: graphs of the script stand for morphemes of spoken Sumerian" (p. 43). Given that Sumerian characters also represented syllables in oral language, it was called a logosyllabary, making Sumerians' cuneiform the world's first syllable (Wolf, 2007). Although some scholars may assume that all writing systems were 
originated from the Sumerian script or genetically related to one another, Sampson (2015) notes that the monogenesis hypothesis is untenable, as "... Chinese writing would be seen as a clear case of a system that developed quite independently of western Asia” (p. 56).

The cuneiform script underwent substantial changes in the outward shape of the graphs over time. Pictograms first used around 3,000 B.C. were rotated $90^{\circ}$ anticlockwise, and then changed into more abstracted glyphs around 2600 B.C., and finally simplified wedge-shaped signs were adopted by Assyrians from the early $1^{\text {st }}$ millennium B.C. until the script's extinction. The systematic $90^{\circ}$ rotation might have had to do with holding the tablet and stylus when writing (Sampson, 2015). Since pressing lines into the clay was not always easy, the shapes of the individual graphs were modified; continuous curved lines were replaced by pointy shapes like a wedge or nail. This led to the name "cuneiform," which simply means "wedgeshaped" (Sampson, 2015).

Cuneiform fulfilled the linguistic needs of the Sumerian, because it was built upon the basic structures of syllables and four vowels, whose forms and meanings could be modified by prefixes and suffixes (Man, 2000). However, the syllabic script bore ambiguities. Sumerian cuneiforms had words and syllables that mixed up spelling, sound, and meaning. As found in other written languages, some words were spelled differently but sounded the same with different meanings (homophones; e.g., in English, too, two, and to; one and won), or spelled the same but differed in meaning (homographs; bat: English, animal bat and baseball bat; bank: financial bank and river bank), or sounded the same but differed in both spelling and meaning (e.g., in English, the 'bill' sound in building or ability; Man, 2000).

\subsubsection{Hieroglyphs}

While Sumerians made inscriptions materialize into a cuneiform system, Egyptians developed their own hieroglyphic system. Given that the Egyptians and Sumerians had massive contact and trade with each other at the time, the Egyptians might have developed their own writing system based on Sumerians' idea of writing (Logan, 2004). Two hundred years after the Sumerian writing system emerged, Egyptian writing hieroglyphs were invented by Semitic tribesmen residing in the Sinai in 3000 B.C. The word hieroglyphs was derived from the ancient Greek for "sacred carvings." Hieroglyphs were first carved on stone and were later written on papyrus with brush and ink (Logan, 2004). Like cuneiforms, Egyptian hieroglyphics were a mixture of logograph and phonogram, although vowels were largely unused and phonographic symbols were used in the sequences of three, two, or one consonant (Sampson, 2015).

Unlike the Sumerian writing system, Egyptian hieroglyphs appeared without a long incubation period. Egyptians developed two parallel writing systems. One was the hieroglyphic system which was formal writing, and the other was hieratic which was a cursive script written on papyrus with brush and ink for written record. Both 
hieroglyphic and hieratic systems adopted pictographic signs with phonetic elements through the addition of foundational signs in a manner similar to those of the Sumerian-Akkadian system (Logan, 2004).

The Egyptians also developed a mixture of uniconsonantal, biconsonantal, and triconsonantal signs to represent all sounds of their spoken language, using approximately 400 signs in total. Although these phonetic signs had the potential to be developed into a more systematic writing system, compared to the logographic cuneiforms, due to being a phonetic alphabet, hieroglyphs did not spread widely nor lived long. The Egyptians might have conservatively restricted their use. Wolf (2007) notes that elites in power probably wanted to keep the complex system to themselves in order to maintain its monopoly on reading and learning and did not want to simplify their writing system. Her observation about the knowledge monopoly is similar to Goody and Watts' (1963) statement on oligoliteracy (i.e., literacy is restricted to the elite). The idea of using the large number of written signs was lost in the course of civilization, however. The Seirites, Semitic tribesmen of the Sinai who mined copper and traded it with the Egyptians, borrowed from the Egyptians the idea of using a small number of consonants to create a vast number of words. Importantly, this was one characteristic of an alphabet. The Seirites were the first people who attempted to write their written signs in an alphabetic manner (Wolf, 2007).

\subsection{The Road to Alphabetic Writing Systems}

Both hieroglyphs and cuneiforms served as the seedbed from which the alphabet spawned (Logan, 2004). The idea of alphabetic writing was borrowed from the Egyptian hieroglyphs by adopting uniconsonantal signs that were the simplest feature of the Egyptian system (Logan, 2004). As briefly mentioned earlier, the hieroglyphic system might have been kept purposely complex by elites so that they could maintain the monopoly of knowledge and privileges (Logan, 2004; Wolf, 2007). Since laypeople were not able to use hieroglyphs as much as they wanted to, the necessity to simplify the writing system became great enough for the powerless to develop a writing system that aligned with their spoken language by adopting uniconsonantal signs which were available at that time. The Proto-Sinaitic alphabet included consonants without vowels.

The word alphabet was originally derived from the Semitic alphabet whose first and second letters were aleph (meaning ox) and bayit (meaning house), respectively. Based on these two graphs, the first two letters of the Greek alphabet, alpha and beta, were created, which in turn became the word alphabet (Logan, 2004; Man, 2000).

There was another alphabetic inscription that was found in three areas in Palestine which was considered 100 or 200 years older than the Sinai inscriptions dating back to 1500 B.C. (Logan, 2004). There were 15 signs of the inscriptions in total, and the representation was extremely pictorial. The level of abstraction was also limited, 
compared to the Sinai inscriptions. For these reasons, the alphabetic inscription in Palestine is not considered as the first alphabet by linguists.

Another type of writing was found on a different continent. Maya hieroglyphs were developed around 300 B.C. as the writing of the Maya civilization of Mesoamerica in modern-day Mexico, El Salvador, Guatemala, Honduras, and Belize. Maya hieroglyphs were a mixture of logograms and phonetic signs representing syllables (Kettunen \& Helmke, 2006). Maya writing primarily used images (pictographs), and was found in stone carvings and scrolls as well as on stucco, wood, pottery, and cloth artefacts. It is known that Maya writing was used until the Spanish conquered the Maya in the sixteenth and seventeenth centuries. The effort to decipher the glyphs is still in progress.

All in all, the Phoenician alphabet was the source of two Semitic alphabets: the early Hebrew alphabet and the Aramaic alphabet. These two alphabets used the Phoenician alphabet at first, but the people developed their own national characters, beginning in 850 B.C. for Hebrew and 750 B.C. for Aramaic, and kept 22 letters of the Phoenician alphabet (Logan, 2004).

The Aramaic script served as the foundation for the birth of many more alphabets. Before the Arabic script was developed, the Neo-Assyrian Empire and later the Persian Empire adopted Aramaic as their official language and script. Gradually, the Aramaic alphabet started to spread to Central Asia and the Indian subcontinent. After the fall of the Persian Empire, the people of these regions developed their own writing systems which were alphabetic, based on the Aramaic alphabet. Thus, the alphabets of India, Afghanistan, and Turkestan were an offspring of Aramaic (Logan, 2004; Wolf, 2007). The Aramaic script also motivated a number of other scripts to be developed in the Middle East, including the Palmyrene script used in Manichean texts from 44 B.C. to A.D. 272, the Syriac script which served as the sacred script of the Assyrian Christian Church from A.D. 200 to the present, and the Nabatean script from 150 B.C. to the sixth century A.D. The Nabatean script directly drove the effort to the development of classical Arabic. The Arabic script, in turn, directly influenced the contemporary Persian script (Logan, 2004; Sampson, 2015).

Although the monogenesis hypothesis may not apply to all scripts available in the world, consensus meets on the claim that all Western alphabetic scripts were derived from the Semitic alphabet. This assumption was made because the creators spoke a Semitic language (possibly Phoenician) and because the properties of Semitic spoken languages influenced certain structural properties of the script (Sampson, 2015). The "Semitic" language is related to the "Afro-Asiatic" or "Hamito-Semitic" family of languages, which are spoken from the Levant westwards to the Atlas and southwards as far as Nigeria, Ethiopia, and Somalia (Sampson, 2015). The two best-known scripts in the Semitic branch are Arabic and Hebrew. The most notable characteristics of the Semitic scripts have consonant graphs but no vowel letters. Although they descended from the Semitic alphabet, many alphabets, including the Roman alphabet, have vowel letters in the inventory. Due to the very reason that Arabic and Hebrew lack vowel graphs, Sampson (2015) claims that it is convenient to reserve the term "Semitic script" for the original Semitic alphabet. 
Hebrew and Arabic scripts remain similar to their common ancestor, except for the outward shapes of the graphs (Sampson, 2015).

\subsubsection{The Greek Alphabet}

When the Greek writing system emerged, the alphabet reached its crescendo of writing. The Greek alphabet was the first writing system that represented the sound system of spoken language, which was the most radical script among all writing systems available until that time (Ong, 1986). The Greek alphabet started to emerge around 900 B.C. and took shape based on the earlier Phoenician alphabet in the Archaic Period (750-480 B.C.; Threatte, 1996). The Greek alphabet was the first full-fledged alphabetic script that had distinct graphs for both consonants and vowels. Before the end of the fourth century B.C., the Greek alphabet existed in many different dialectal variations. When the Euclidean alphabet used 24 letters in the order from alpha to omega, it became the standard that has carried on until today. This became the ancestor of the Latin and Cyrillic scripts. The Greek alphabet originally had a single case for each letter, but the uppercase and lowercase letters were developed during the modern era.

\subsubsection{True Alphabet}

Man (2000) asserts that one of the major roots of Western identity can be found in the alphabet. The alphabet manifests the essence of simplicity (Man, 2000). At the heart of alphabetic writing systems are phonemes as the minimal linguistic unit. Goody and Watts (1963) put forth an interesting linguistic claim that each culture's phonemic inventory is the most extreme and the most universal example of cultural selection. Their assertion was based on the notion that the number of sounds of which human beings can articulate is vast but gets narrowed down over time to accord with their language. Alphabetic scripts incorporate the universal byproduct of cultural selection (that is, phonemes) effectively in their writing systems by using about 40 of the phonemes in each writing system. With the inventory of about 40 phonemes, alphabetic writing systems can have a large number of syllables. For example, English has more than 10,000 syllables using 36 to 44 phonemes; Spanish has 25 or 26 phonemes [5 vowels and $19(+1)$ consonants including allophones and diphthongs]; and there are 39 phonemes in French [17 vowels and $20(+2)$ vowels] as well as 45 phonemes in German (17 vowels and 25 consonants). All alphabetic writing systems conform to the alphabetic principle, meaning that letters represent the sounds of oral language (not morphemes like Chinese characters) and that multiple phonemes are combined to form a syllable in the word.

Although the feasibility of using phonemes in the writing system is universal, not all languages utilize phonemes as the minimal sound unit in the system. For 
example, Chinese characters are not composed of phonemes but represent syllables. Japanese is the same. Due to the combinatory rule for phonemes to produce syllables, the numbers of syllables are far greater in alphabetic scripts than those of syllabic scripts, such as Chinese and Japanese, which have 400 syllables without considering tones and about 100 syllables, respectively (Taylor \& Taylor, 2014). The system of the graphic representations of alphabetic languages allows users to learn to read and write easily because learners do not need to memorize the large number of characters through rote memorization as in when learning to read in Chinese.

\subsection{Chinese Writing System}

Although the monogenetic hypothesis that all writings evolved from the same genesis was advocated by Gelb (1952), Sampson (2015) questions the monogenesis, thinking that Chinese characters might be developed independently of other writing systems. The record of the outset of Chinese characters is lost from the archaeological record or has not been found yet (Bagley, 2004). Since the two ends of Asia are connected by land, cultural borrowing from each other can be assumed. Hence, it is still debatable whether Chinese characters were developed in a completely independent manner or not. The earliest Chinese writing samples were found in the form of divination texts that were carved on bones and turtle shells in approximately 1320 B.C. (Bagley, 2004; Siqi, 2018). Although the carved word signs included names, dates, and items of sacrifice, archaeologists still could not determine what preceded the carved word signs. The shape of the characters and the context in which they were used indicated a well-developed scribal class, which suggested a complex society the Chinese established in antiquity. Since a more in-depth coverage of Chinese characters, along with Japanese Kanji, is reserved to Chapter 5, I keep the discussion of the Chinese logographic system short in this chapter.

\subsection{From Numeracy to Literacy}

This section serves as a chapter summary in relation to the evolution from numbers to written signs. In the time of hunters and gatherers, the tally and token systems remained largely static as they fulfilled the notational needs at the time. When agricultural civilization emerged, socioeconomic situations became more complex and began to change gradually. The tally and token systems grew elaborated and were modified to meet the demands of more sophisticated commercial transactions and trades in a new form of society (Logan, 2004). When the urbanization of Sumer at Uruk gradually took place between 3350 B.C. and 3100 B.C., tokens were used for tax payments, the distribution of rations, and the control of irrigation systems which were essential for all agriculture (Logan, 2004; Sampson, 2015). The coercive 
taxation and redistribution systems forced the token system to go through a major transformation. It started to function as an administrative tool for the political and commercial control of agriculture. It also yielded chain reactions in new information processing associated with abstract numeration, logographic writing, and phonetic coding (Logan, 2004; Sampson, 2015).

Urbanization required more complex accounting systems due to the increased number of social interactions and items to be enumerated. Such necessity yielded the systematic ways of encoding, which led to the repeated use of the small number of signs by combining the signs. This led to the invention of alphabetic writing, which uses the small number of signs to create the large number of words under the blending rule of multiple signs. This was very akin to the alphabetic principle which means that the minimal unit corresponds to phonemes and that graphs group together to form a syllable.

The emergence of a new writing system is a reflection of the complexity and necessity that users came across at the time. As sociocultural situations became complicated, the users encountered a new level of complexities involved in their lives. The advent of the first primitive alphabet writing, known as the Proto-Sinaitic script, materialized in the Sinai and Canaan in the first half of the second millennium B.C. (Logan, 2004). This was the cornerstone for the development of the more full-fledged Greek alphabet that included both consonants and vowel graphs. This chapter has reviewed a historical trajectory of writing systems in different continents as a part of the introductory part of the book, PART I. Chapters 4 and 5 in PART II provide more detailed accounts of the characteristics of the alphabet and the development of Chinese characters.

Open Access This chapter is licensed under the terms of the Creative Commons Attribution 4.0 International License (http://creativecommons.org/licenses/by/4.0/), which permits use, sharing, adaptation, distribution and reproduction in any medium or format, as long as you give appropriate credit to the original author(s) and the source, provide a link to the Creative Commons license and indicate if changes were made.

The images or other third party material in this chapter are included in the chapter's Creative Commons license, unless indicated otherwise in a credit line to the material. If material is not included in the chapter's Creative Commons license and your intended use is not permitted by statutory regulation or exceeds the permitted use, you will need to obtain permission directly from the copyright holder.

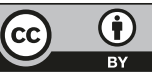

